\title{
Asymmetrical Half-Join Method on Dual Vision Face Recognition
}

\author{
Edy Winarno ${ }^{1}$, Imam Husni Al Amin², Wiwien Hadikurniawati ${ }^{3}$
}

Faculty of Information Technology, Universitas Stikubank, Semarang, Indonesia

\begin{tabular}{|c|c|}
\hline Article Info & ABSTRACT \\
\hline & \multirow{9}{*}{$\begin{array}{l}\text { This research proposes a model of face recognition using the method of } \\
\text { joining two face images from left and right lens from a stereo vision camera } \\
\text { namely half-join method. Half-join method is used in face image } \\
\text { normalization processing. The proposed half-join method is a face images } \\
\text { joining model, which is called asymmetrical half-join. In asymmetrical half- } \\
\text { join method, a RoI (region of interest) of face image from left and right } \\
\text { lenses are provided based on axis center of each eye in eye detection. The } \\
\text { cropping of face image from asymmetrical half-join model has different } \\
\text { width depends on eyes coordinate location. The proposed system shows that } \\
\text { the asymmetrical half-join method can produce a better of face recognition } \\
\text { rate. The experimental results show that the asymmetrical half-join method } \\
\text { has a better recognition rate and computation time than single vision method } \\
\text { and symmetrical half-join method. }\end{array}$} \\
\hline Received May 9, 2017 & \\
\hline Revised Jun 26, 2017 & \\
\hline Accepted Jul 19, 2017 & \\
\hline Keyword: & \\
\hline Face recognition & \\
\hline Asymmetrical & \\
\hline Half-join & \\
\hline Stereo vision & \\
\hline
\end{tabular}

Copyright $@ 2017$ Institute of Advanced Engineering and Science. All rights reserved.

\section{Corresponding Author:}

Edy Winarno,

Faculty of Information Technology,

Universitas Stikubank Semarang,

J1. Tri Lomba Juang No. 1 Semarang, 50241, Jawa Tengah, Indonesia.

Email: edywin@edu.unisbank.ac.id

\section{INTRODUCTION}

In face recognition system, the use of a camera as a face image acquisition method plays an important role. The camera is used to capture the image of the face using face detection which then is processed using normalization techniques, feature extraction and classification to identify a face. In the face recognition system, the camera is used to generate facial image data to be processed in the image of twodimensional (2D) or three-dimensional images (3D). Generally, to produce a two-dimensional image on face recognition we still use a single vision camera for the acquisition. In the $3 \mathrm{D}$ image acquisition models, generally we use stereo vision camera or other image acquisition devices such as scanner etc.

Stereo vision camera can also be used to generate both $2 \mathrm{D}$ and $3 \mathrm{D}$ images simultaneously. A combination of 2D and 3D facial image is used for face recognition models. Research that combines 2D and $3 \mathrm{D}$ facial images in a face recognition system is applied by [1] and [2]. In some studies of face recognition system, stereo vision camera is widely used in face recognition processing to produce a 3D image. This model is used to reconstruct points of facial image, and then used to generate a 3D facial image in face recognition. Some studies using stereo vision camera is used to generate a $3 \mathrm{D}$ image reconstruction in facial recognition models, as conducted by [3]-[5].

From several previous studies, the use of stereo vision camera has not been widely used to produce a joining of 2D facial image from the right and left cameras. Stereo vision camera is used to produce a model of face recognition in robotic vision systems. The use of stereo vision camera on a robotic vision system is used to perform a face recognition model and to measure the distance between the face and the camera. Stereo vision camera can also be used to determine the distance of a person's face using a triangulation method that utilizes a pair of cameras to determine the distance as conducted by [6] and [7]. 
Although some studies still use stereo vision camera to reconstruct a 3D image for face recognition and to determine the distance of the face, but there is still a research-gap in research of 2D image that can be elaborated using stereo vision camera. In this research, we create a model to determine the correlation of stereo vision camera that elaborates the model of face recognition using a $2 \mathrm{D}$ image. Face recognition system using 2D image requires less computing time than the facial recognition using 3D image [8]-[12].

Face recognition using stereo vision camera will produce two face images with different angles. Both of face image has a detailed face image on each side. Left-side face image on the left camera will look more detailed than the right-side facial image. Right-side face image on the right camera will also be seen in more detail than the left-side face image. The image combination between left-face image and right-face image on stereo vision camera can be used to anticipate variations of pose in face recognition process [13].

Regarding the issue of illuminance, stereo vision camera can produce images with better illuminance levels on each of the camera lens. Left-side facial image on the left camera will get better illumination than the right-side face image. Right-side face image on the right of the camera will also get better illumination than the left-side facial image. The left camera and the right camera on stereo vision camera can maximize the level of illuminance on each side of the face image, as well as anticipating variation of illuminance.

This study proposes a model of 2D facial image acquisition from two camera lenses on stereo vision. Face image normalization is conducted using preprocessing techniques and a facial image joining method namely half-join [13]. Half-join is a joining model between a half of left face image from left-camera with a half of right face image from right-camera using stereo vision camera. The proposed method of halfjoin that used in this study is a face images joining model, namely asymmetrical half-join. We use normalization process of the face image using preprocessing and asymmetrical half-join method. In asymmetrical half-join, we use RoI (region of interest) of face image from left and right lenses that provided from axis center of each eye in eye detection. In the experiment results, we compare the recognition rate using the images from single vision camera, symmetrical half-join [13] and asymmetrical half-join.

\section{RESEARCH METHOD}

Asymmetrical half-join method is inspired by the model of the human perspective using two eyes to see and identify a person. Both of the human eyes are represented using a dual camera vision that has left and right lenses. The use of two images in face recognition is used to implement the use of the features of the two different views simultaneously. This method combines the left half of the image and the right half of the image into an image that is ready to be extracted on its features. Face recognition process using asymmetrical half-join method can be illustrated in Figure 1.

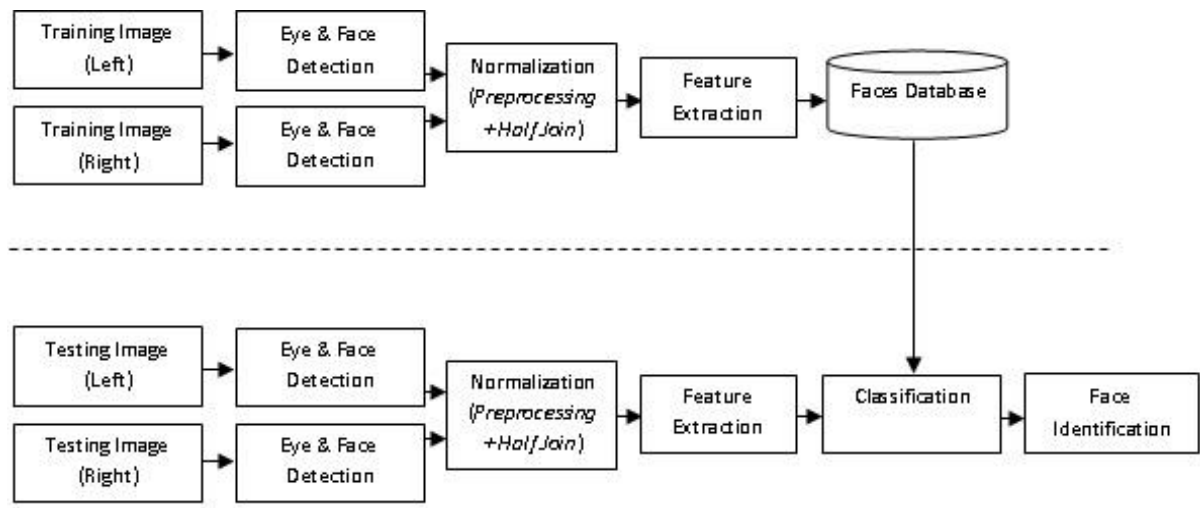

Figure 1. Face Recognition Using Asymmetrical Half-join Method

In image acquisition process, face image is taken by the left and right lenses in stereo vision camera. This process produces two RGB image size of 640 x 480 pixels. The next step is to detect faces and eyes used to ascertain whether there is a face in the image of the acquisition. Half-join system will work if both of the left and right lenses capture human face images. Half-join processing will not work if only one lens that captures the image of a face. Half-join processing will repeat until the acquisition of the lenses capture the image of a face.

IJECE Vol. 7, No. 6, December 2017 : 3411 - 3420 
In asymmetrical half-join method, eye detection is used to locate the axis on the left and right eye in the image. The position of the eye is used as a determinant of the location of the cutting area on the face. The position of the axis is a relative position of the raw image acquisition results. The next axis position is recalculated in order to illustrate the relative position of the face image.

Asymmetrical half-join method requires the calculation to determine the point-cutting of the image. Point-cutting of the image is not in the middle of the image. It will produce the images with different width. The left side area of the face image on the left lens is then combined with the right side area of the image on the right lens. The result of the face image combination has a different width with face image source. It will be resized to the same width as the face image source.

Asymmetrical half-join is a method used to divide the width of the face image on the left and right lens camera with unequal size. In this method, Haar eye detection [14] was used to determine RoI of eye image. The first step in the method of asymmetrical half-join was to determine the width of the face image ( $w$ / width) in pixels. This method requires the midpoint of the left eye (le) and right eye (re) to determine the point of cutting $(c)$. The point of cutting is the sum of the midpoint of the left and right eyes. Then, divided by $2(c=(l e+r e) / 2)$.

Image cutting point is calculated on the left and right images, so that the cutting point of the left and right images will be different. Face image of the left lens of the camera is the cropping area between the left point $(x=0)$ and the pixels before the cutting point $(x=c-1)$. Face image of the right lens camera is the cropping area between the cutting point $(x=c)$ and the right-most point of the image $(x=w-1)$. Both of face images are then combined and resized similar to the size of the facial image $(w)$.

The first step in the method of asymmetrical half-join is to determine the axis point of cutting point ( cla ). This cutting point is the midpoint between the point of the left eye (le) and right eye point ( $r e$ ) on the face image detection result from left lens of the camera, as shown in Equation (1).

$$
c l a=\frac{l e+r e}{2}
$$

The second process is to determine the axis of cutting point ( $\mathrm{cra}$ ). $\mathrm{cra}$ is the midpoint between the point of the left eye ( $l e$ ) and right eye point ( $r e$ ) on the face image detection results of the right lens of the camera, as shown in Equation (2).

$$
\text { cra }=\frac{l e+r e}{2}
$$

The next step is to cut the face image detection result of the left lens of the camera $a h j_{l e f t}$. Cutting process is done in the area of the face $(\mathrm{x}=0)$ to $(\mathrm{x}=c l a-1)$ as shown in Equation (3).

$$
\operatorname{ahj}_{l e f t}=\sum_{x=0}^{c l a-1} c l a
$$

The same process is also performed on the face image detection results of the right lens of the camera $a h j_{r i g h t}$. Cutting process is done in the area of the face $(\mathrm{x}=c r a)$ to $(\mathrm{x}=w-1)$ as shown in Equation (4).

$$
{ }^{a h j_{\text {right }}}=\sum_{x=c r a}^{w-1} w
$$

The result of a join between the face image cropped on the left lens of the camera $a h j_{l e f t}$ and the face image cropped on the right lens of the camera $a h j_{\text {right }}$ is shown in Equation (5) and (6).

$$
\begin{aligned}
& a h j=a h j_{\text {left }}+a h j_{\text {right }} \\
& a h j=\sum_{x=0}^{c l a-1} c l a+\sum_{x=c r a}^{w-1} w
\end{aligned}
$$


Face image combining result has different pixel size than the original size. Resizing process is needed to restore it. Asymmetrical half-join method has two additional RoI determining step to get a partial area of the face on the left and right lens. RoI is a vertical rectangular area. Each RoI has different width but has the same height. This process also seeks the coordinate of the top left, top right, bottom left and bottom right. The coordinates, width and height of the image used in the calculation are the coordinates of the RoI during face detection.

The midpoint of the face of the asymmetrical half-join is the midpoint between the left and right eyes. Eye detection using Haar [14] was used to detect the location of the left and right eyes. Eye detection is used to obtain the coordinates of the point $\left(x_{a}, y_{a}\right)$ on the top left corner of the left eye image, the coordinates of points $\left(x_{b}, y_{a}\right)$ on the top left corner of the right eye image, the width $(w)$ and height $(h)$.

The midpoint of the face ( $c l a$ and $c r a$ ) is calculated by dividing the sum of the midpoint of the eye. The midpoint of the left eye is the point of the upper left corner of left eye $\left(x_{a}, y_{a}\right)$ plus half the width of the left eye image $\left(1 / 2 w_{1}\right)$. The midpoint of the right eye is the point of the upper left corner of right eye $\left(x_{b}, y_{a}\right)$ plus half the width of the right eye image $\left(1 / 2 w_{2}\right)$. Determination of coordinates of left and right eyes on the asymmetrical half-join can be illustrated in Figure 2.

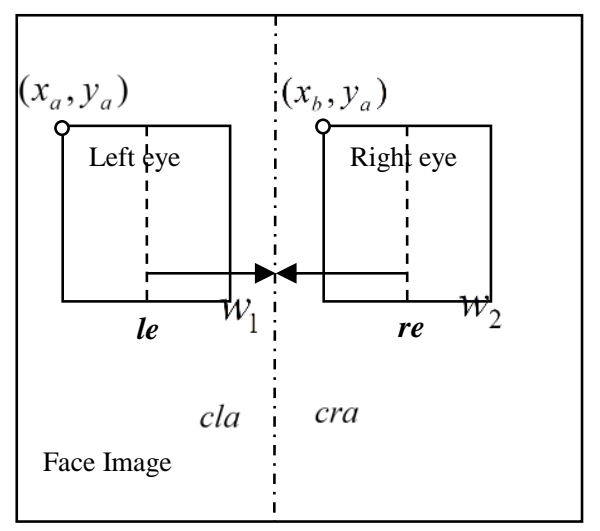

Figure 2. The Coordinates of Left and Right Eyes on Asymmetrical Half-join

Coordinates of the starting point $\left(x_{a}, y_{a}\right),\left(x_{b}, y_{a}\right), w_{1}$ and $w_{2}$ is used to obtain the value of the midpoint of the left eye ( $l e)$, the midpoint of the right eye ( $r e$ ) and the middle of cutting point in the face image ( $c l a$ and $c r a$ ). le is calculated using Equation (7).

$$
l e=x_{a}+1 / 2 w_{1}
$$

To determine the value of $r e$ is solved using Equation (8).

$$
r e=x_{b}+1 / 2 w_{2}
$$

The midpoint of the face ( $c l a$ dan cra ) is calculated using Equation (9) and (10).

$$
\begin{aligned}
& \text { cla }=1 / 2(l e+r e) \text { or } \operatorname{cra}=1 / 2(l e+r e) \\
& c l a=1 / 2\left(x_{a}+1 / 2 w_{1}+x_{b}+1 / 2 w_{2}\right) \text { or } c r a=1 / 2\left(x_{a}+1 / 2 w_{1}+x_{b}+1 / 2 w_{2}\right)
\end{aligned}
$$


cla and cra are used as the cutting point to divide RoI of face images on left and right area. Both of RoI face images are then combined to produce a face image that will be used in the feature extraction process. 2.1. RoI of left half-face

$\left(x_{0}, y_{0}\right)$ and $\left(x_{0}, y_{1}\right)$ are the coordinates of the image pixel column. $\left(x_{1}, y_{0}\right)$ and $\left(x_{1}, y_{1}\right)$ are determined based on the cutting point ( $c l a$ ) in the image, so $x_{1}$ can be discovered. $y_{1}$ is determined based on the height $(h)$ in the image. RoI partial image of the left face of the left lens can be illustrated in Figure 3.

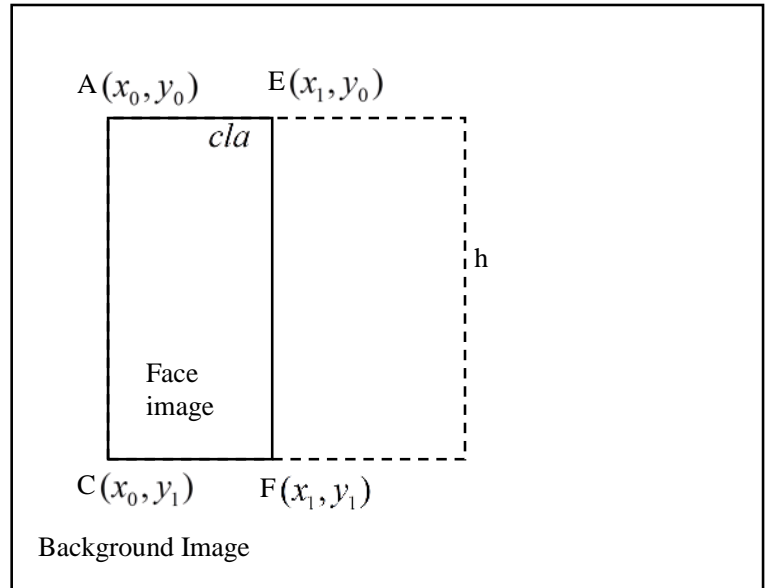

Figure 3. RoI Partial Image of The Left Face of The Left Lens

$$
\begin{aligned}
& x_{1} \text { and } y_{1} \text { is calculated using Equation (11) and (12). } \\
& x_{1}=x_{0}+(\text { cla }-1) \\
& y_{1}=y_{0}+(\mathrm{h}-1)
\end{aligned}
$$

A, E, C and F are the RoI coordinate points of the partial image of the left face on the left lens. It can be calculated using Equation (13) - (16).

$$
\begin{aligned}
& \mathrm{A}=\left(x_{0}, y_{0}\right) \\
& \mathrm{E}=\left(x_{0}+(c l a-1), y_{0}\right) \\
& \mathrm{C}=\left(x_{0}, y_{0}+(\mathrm{h}-1)\right) \\
& \mathrm{F}=\left(x_{0}+(c l a-1), y_{0}+(\mathrm{h}-1)\right)
\end{aligned}
$$

RoI at coordinates A, E, C and F is then combined with a partial image of the right face of the right lens of the camera. This image is a face image of asymmetrical half-join.

\subsection{RoI of right half-face}

$\left(x_{0}, y_{0}\right)$ and $\left(x_{0}, y_{1}\right)$ are the primary coordinate points in the column pixel of image. These coordinate points are used to determine the ROI of the right face image of the right lens. $\left(x_{1}, y_{0}\right)$ and $\left(x_{1}, y_{1}\right)$ are determined by the cutting point $(\mathrm{cra}) .\left(x_{2}, y_{0}\right)$ and $\left(x_{2}, y_{1}\right)$ are determined by the width $(w)$ 
and height $(h)$ of the face image, and it is calculated from the coordinates of $\left(x_{0}, y_{0}\right)$ and $\left(x_{0}, y_{1}\right)$. RoI partial image of the right face of the right lens can be illustrated in Figure 4.

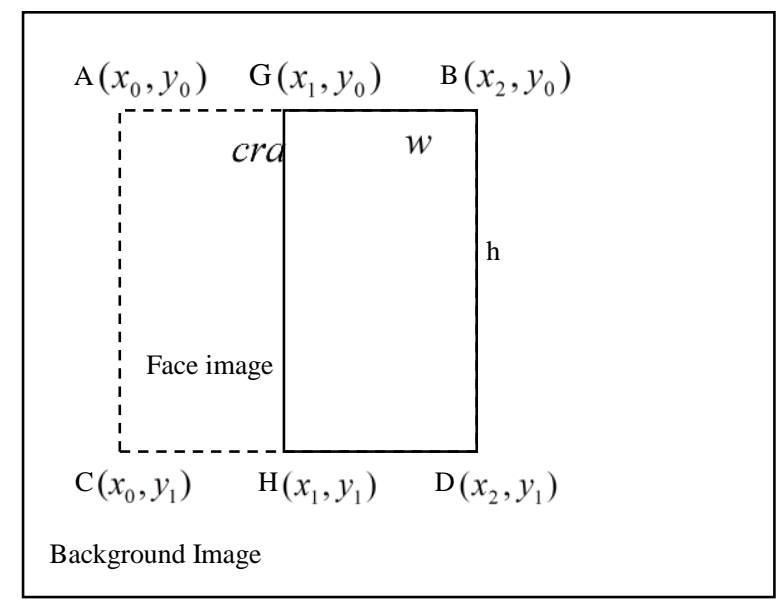

Figure 4. RoI Partial Image of The Right Face of The Right Lens

$$
\begin{aligned}
& x_{1}, y_{1} \text { and } x_{2} \text { is calculated using Equation (17), (18) and (19). } \\
& x_{1}=x_{0}+\operatorname{cra} \\
& y_{1}=y_{0}+(\mathrm{h}-1) \\
& x_{2}=x_{0}+(\mathrm{w}-1)
\end{aligned}
$$

G, B, H and D are the RoI coordinate points of the partial image of the right face on the right lens. It is calculated using Equation (20)-(23).

$$
\begin{aligned}
& \mathrm{G}=\left(x_{0}+c r a, y_{0}\right) \\
& \mathrm{B}=\left(x_{0}+(\mathrm{w}-1), y_{0}\right) \\
& \mathrm{H}=\left(x_{0}+c r a, y_{0}+(\mathrm{h}-1)\right) \\
& \mathrm{D}=\left(x_{0}+(\mathrm{w}-1), y_{0}+(\mathrm{h}-1)\right)
\end{aligned}
$$

RoI at coordinates $\mathrm{G}, \mathrm{B}, \mathrm{H}$ and $\mathrm{D}$ is then combined with RoI at coordinates A, E, C and F. It is resized to 160x160 pixel of image. This image is a face image of asymmetrical half-join.

\section{RESULTS AND ANALYSIS}

This study uses the Viola-Jones face detection. Each left and right lenses of a stereo vision camera is used to take a raw image with the background image size of 640x480 pixels and the detected face image. Width and height of the face image depends on the distance between the face and the camera. The face image detected on the background image is marked with the coordinates of the position of the face image RoI. The face image RoI is then stored into the variable storage face image. The face image is then normalized using preprocessing and half-join method.

In preprocessing, the cutting process is conducted to take the face image and it is converted into grayscale. The grayscale image is then adjusted using the resizing process to get a face image size of 
160x160 pixels. The adjustment of contrast and brightness is conducted using the histogram equalization. It is used to produce the better face image quality. Image results in each of the camera lens is then processed using the method of asymmetrical half-join. The examples of some RoI face image preprocessing results on a stereo vision camera in some poses and taken at the same time as seen in Figure 5.

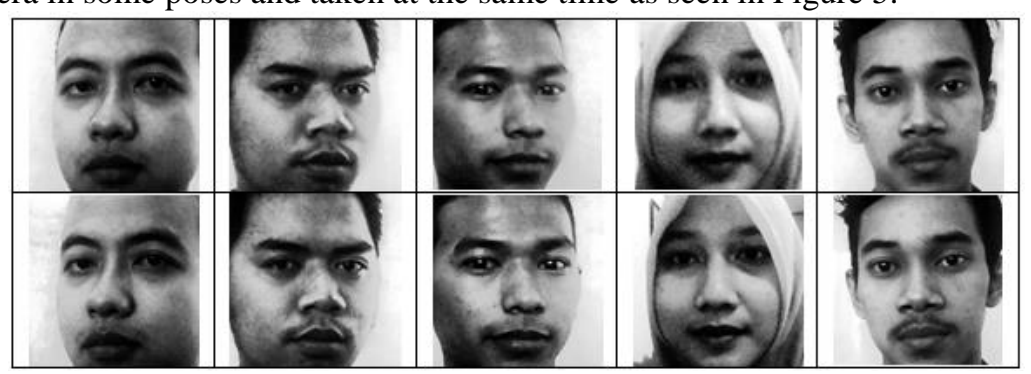

Figure 5. Face Image Preprocessing Results on A Stereo Vision Camera, (above) The image of The Left Lens, (bottom) The Image of The Right Lens

Left eye detection and right eye detection is used in asymmetrical half-join method to determine the cropped area of face image. The left face image is produced by coordinates $\mathrm{A}\left(x_{0}, y_{0}\right), \mathrm{E}\left(x_{1}, y_{0}\right), \mathrm{C}$ $\left(x_{0}, y_{1}\right)$ and $\mathrm{F}\left(x_{1}, y_{1}\right)$. The right one is produced by $\mathrm{G}\left(x_{1}, y_{0}\right), \mathrm{B}\left(x_{2}, y_{0}\right), \mathrm{H}\left(x_{1}, y_{1}\right)$ dan $\mathrm{D}\left(x_{2}, y_{1}\right)$. The size of all of the face images has different width and height.

The partial image of the left face of the left lens and the partial image of the right face of the right lens are then combined to produce a face image of asymmetrical half-join. The examples of face images of asymmetrical half-join with different size is seen in Figure 6.

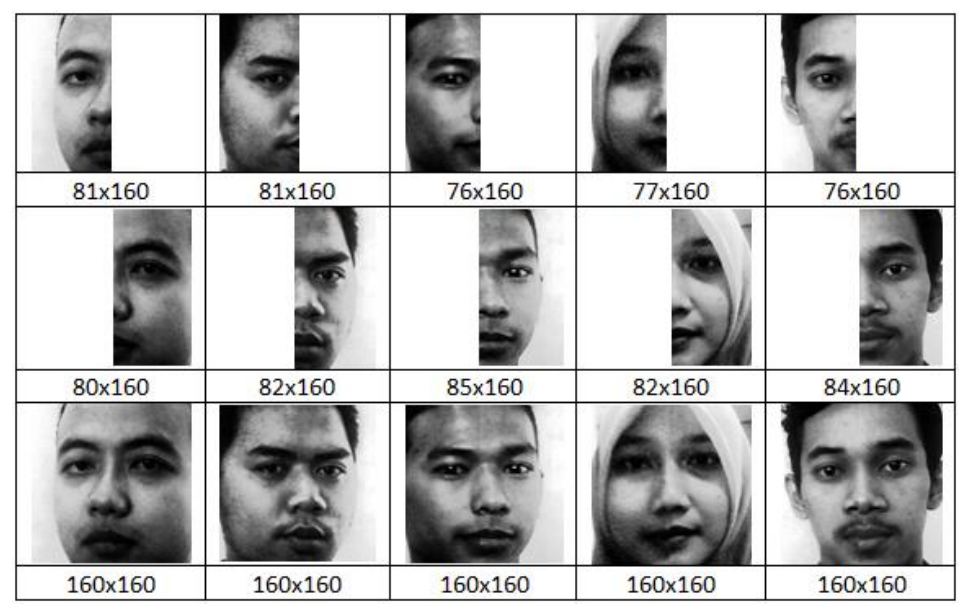

Figure 6. The Face Image of Asymmetrical Half-Join, (above) The Partial Image of The Left Face of The Left Lens, (midle) The Partial Image of The Right Face of The Right Lens, (bottom) Combined Model

In this research, the test is conducted by comparing between dataset from single vision face image, symmetrical half-join face image ( dividing size of face image symmetrically) [13] and asymmetrical halfjoin face image. The examples of all of the images is seen in Figure 7. 


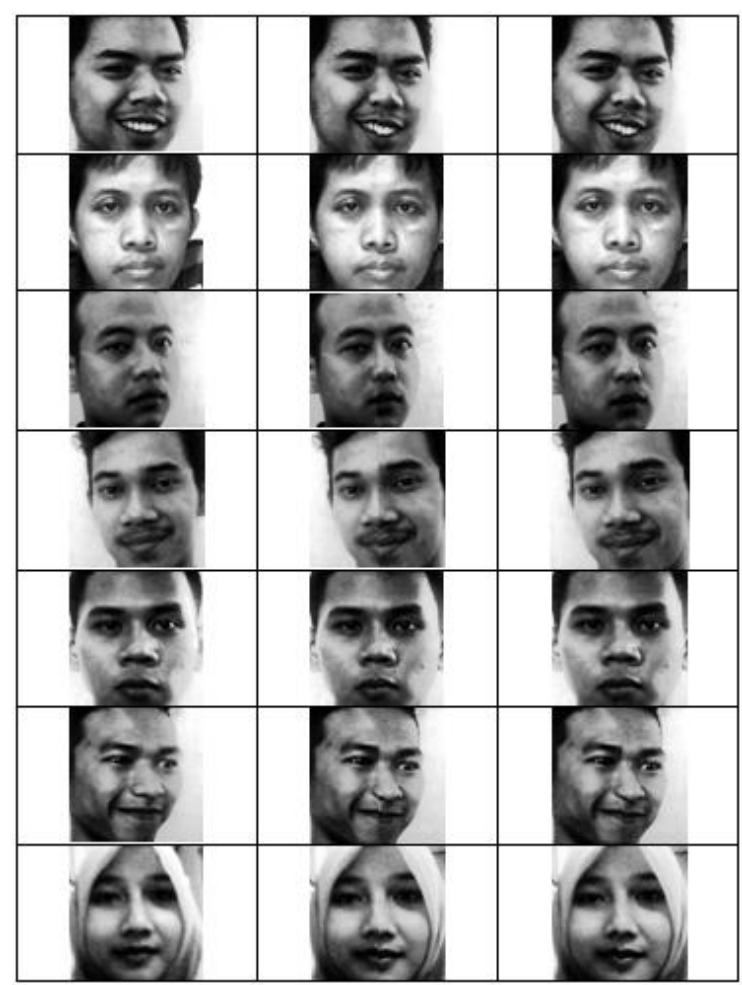

Figure 7. Face Images of Testing, (left) Single Vision Face Image, (midle) Face Image of Symmetrical HalfJoin, (right) Face Image of Asymmetrical Half-Join

Test face images are taken from 40 people each were taken 10 times. The results are 400 single vision face images, 400 symmetrical half-join face images and 400 asymmetrical half-join face image. The testing is conducted by comparing each test images with the training images at each method. It is also conducted by comparing single vision test images with single vision training images, symmetric half-join test images with symmetric half-join training images and asymmetrical half-join test images with asymmetrical half-join training images on real time conditions. The test results show that the method of single vision testing has an average accuracy of $96 \%$, on a symmetrical half-join method has an average accuracy of $93.25 \%$ and asymmetrical half-join method has an average accuracy of $97.5 \%$. The average of face recognition rate using a single vision, symmetrical and asymmetrical half-join is illustrated in Figure 8.

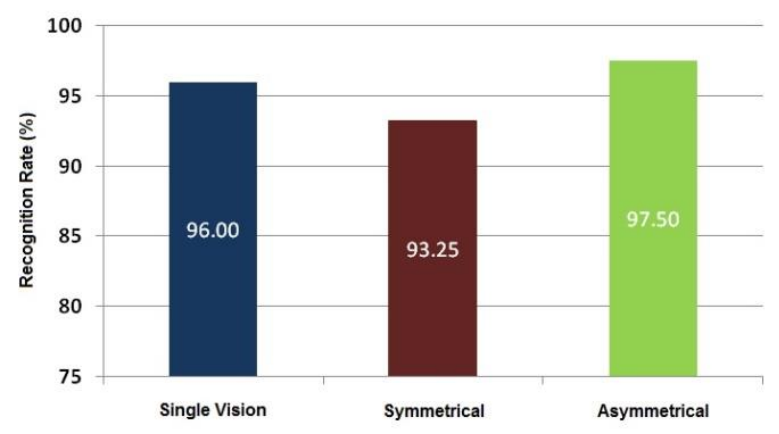

Figure 8. Face Recognition Rate (\%)

The measurement of computation time of face recognition is conducted simultaneously with the testing of face recognition rate on the method of single vision, symmetrical and asymmetrical half-join. The tests produced an average of computation time of face recognition on each method. The test results show that 
the single vision method has an average computation time of $45.49 \mathrm{~ms}$, symmetrical half-join method has an average computation time of $47.80 \mathrm{~ms}$ and asymmetrical half-join has an average computation time of 45.99 ms. The computation time of face recognition on each method is illustrated in Figure 9.

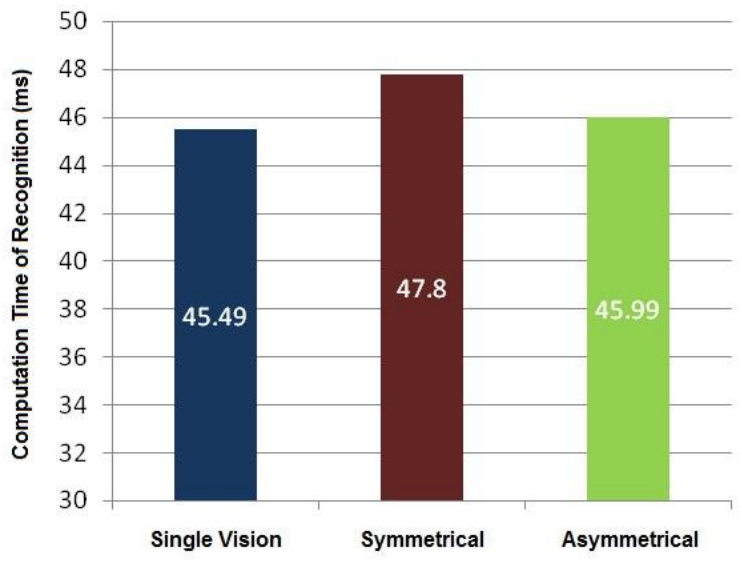

Figure 9. The Computation Time of Face Recognition (millisecond)

\section{CONCLUSION}

Generally, the big problem in face recognition system is how to solve the variation of poses and the variation of illuminance to get the best recognition rate and to decrease recognition time. In this study we use two lenses on stereo vision camera. Generally, a stereo vision camera is used in a face recognition system to reconstruct dots of face then to produce a three-dimensional (3D) face image. However, the process of face recognition using 3D images require more recognition time than the use of two-dimensional (2D) face images. This research proposes a model of face recognition using the method of joining two face images from left and right lens from a stereo vision camera namely asymmetrical half-join method. Asymmetrical half-join method is used in face image normalization processing. In asymmetrical half-join method, a RoI of face image from left and right lenses are produced based on center axis of each eye from eye detection. The cropping face image from asymmetrical half-join model has different wide of cropping depend on eyes coordinate location.

Based on the experiments, the proposed system shows that the asymmetrical half-join method is able to produce average $97.5 \%$ of face recognition rate and has an average computation time of face recognition of $45.99 \mathrm{~ms}$. The asymmetrical half-join method has a better accuracy than face recognition system using single vision method and symmetrical half-join method. This study shows that the stereo vision camera has a capability to produce two of 2D face images from each lens that is able to be elaborated with 2D face image recognition technique and also to produce high recognition rate and short recognition time.

\section{REFERENCES}

[1] G. P. Kusuma and C. S. Chua, "PCA-based image recombination for multimodal 2D+3D face recognition," Image and Vision Computing, vol/issue: 29(5), pp. 306-316, 2011.

[2] A. Moeini, et al., "Expression-Invariant Face Recognition via 3D Face Reconstruction Using Gabor Filter Bank from a 2D Single Image," in Pattern Recognition (ICPR), 2014 22nd International Conference on, pp. 4708-4713, 2014.

[3] H. M. Rara, et al., "Distant face recognition based on sparse-stereo reconstruction," in Image Processing (ICIP), 2009 16th IEEE International Conference on, pp. 4141-4144, 2009.

[4] E. Temizkan and H. S. Bilge, "Shape index based 3d face recognition using photometric stereo," in 2011 IEEE 19th Signal Processing and Communications Applications Conference (SIU), pp. 454-457, 2011.

[5] M. A. Abdelrahman, et al., "A passive stereo system for 3D human face reconstruction and recognition at a distance," in 2012 IEEE Computer Society Conference on Computer Vision and Pattern Recognition Workshops, pp. 17-22, 2012.

[6] C. Holzmann and M. Hochgatterer, "Measuring distance with mobile phones using single-camera stereo vision," Distributed Computing Systems Workshops (ICDCSW), 32nd International Conference on, IEEE, pp. 88-93, 2012.

[7] E. Winarno, et al., "Development of Face Recognition System and Face Distance Estimation Using Stereo Vision Camera," Journal of Theoretical and Applied Information Technology, vol/issue: 67(3), pp. 652-657, 2014. 
[8] J. Harguess and J. K. Aggarwal, "A case for the average-half-face in 2D and 3D for face recognition," in Computer Vision and Pattern Recognition Workshops, 2009. CVPR Workshops 2009. IEEE Computer Society Conference on, pp. 7-12, 2009.

[9] S. Zafeiriou, et al., "Face recognition and verification using photometric stereo: The photoface database and a comprehensive evaluation," Information Forensics and Security, IEEE Transactions on, vol/issue: 8(1), pp. 121135, 2013.

[10] E. Winarno, et al., "Improved Real-Time Face Recognition Based On Three Level Wavelet DecompositionPrincipal Component Analysis And Mahalanobis Distance," Journal of Computer Science, vol/issue: 10(5), pp. 844-851, 2014.

[11] D. Sridhar and I. M. Krishna, "Face Recognition Using Two Dimensional Discrete Cosine Transform, Linear Discriminant Analysis and K Nearest Neighbor Classifier," IAES International Journal of Artificial Intelligence, vol/issue: 1(4), pp. 161, 2012.

[12] S. N. Randive and A. B. Gonde, "A novel approach for face recognition using fusion of local gabor patterns," International Journal of Electrical and Computer Engineering (IJECE), vol/issue: 2(3), pp. 345, 2016.

[13] E. Winarno, et al. "Face Recognition Based on Symmetrical Half-Join Method using Stereo Vision Camera," International Journal of Electrical and Computer Engineering, vol/issue: 6(6), pp. 2818-2827, 2016.

[14] P. Viola and M. Jones, "Rapid object detection using a boosted cascade of simple features," in Proceeding IEEE Conference on computer vision and pattern recognition 2001, pp. 1-9, 2001. 